\title{
The Casimir Check for the Algebraic Matrices of the Configuration $(d+s)^{n} p^{*}$
}

\author{
Charles Roth**
}

(February 23, 1971)

\begin{abstract}
By using the eigenvalues of the Casimir operator for the group $S U_{3}$, checking parameters were obtained for 12 electrostatic interaction matrices of the configuration $(d+s)^{n} p$.

Key words: Casimir operator, checking parameters, $(d+s)^{n} p, S U_{3}$.
\end{abstract}

\section{Introduction}

The algebraic matrices of $(d+s)^{n} p$ comprise the electrostatic and spin-orbit interaction matrices of the configurations $d^{n} p, d^{n-1} s p$ and $d^{n-2} s^{2} p$, the matrices of the correction parameters representing two and three body interactions of the core $d$ electrons, as well as the matrices of the interactions between configurations. The energy matrix (for a particular $n$ ) is then a linear combination of these matrices, the coefficients of which are parameters usually obtained empirically by fitting the experimental levels to the eigenvalues of the energy matrix. The following lists these parameters and gives their significance. Unprimed quantities denote the configuration $d^{n} p$, primes denote $d^{n-1} s p$ and double primes $d^{n-2} s^{2} p$.

$A, A^{\prime}, A^{\prime \prime}, \quad$ the heights of the configurations

$B, B^{\prime}, B^{\prime \prime} \quad$ linear combinations of the Slater parameters

$F^{2}(d d)$ and $F^{4}(d d)$

$B=1 / 441\left[9 F^{2}(d d)-5 F^{4}(d d)\right]=F_{2}(d d)-5 F_{4}(d d)$

$C, C^{\prime}, C^{\prime \prime}$

multiples of the Slater parameter $F^{4}(d d)$

$G_{d s}^{\prime}$

$$
C=5 / 63 F^{4}(d d)=35 F_{4}(d d)
$$

the parameter of the $d-s$ interaction in the configuration $d^{n-1} s p$

$$
G_{d s}^{\prime}=1 / 5 G^{2}(d s)
$$

$F_{2}, F_{2}^{\prime}, F_{2}^{\prime \prime} \quad$ parameters of the direct part of the $d-p$ interaction

$$
F_{2}=1 / 35 F^{2}(d p)
$$

$G_{1}, G_{1}^{\prime}, G_{1}^{\prime \prime} \quad$ parameters of the exchange part of the $d-p$ interaction

$$
G_{1}=1 / 15 G^{1}(d p)
$$

AMS Subject Classification: Primary 81A66.

*An invited paper. This work was supported by the National Bureau of Standards.

**Present address: Department of Mathematics, McGill University, Montreal, Canada.

${ }^{1}$ Figures in brackets indicate the literature references at the end of this paper. 
$G_{3}, G_{3}^{\prime}, G_{3}^{\prime \prime} \quad$ parameters of the exchange part of the $d-p$ interaction

$$
G_{3}=3 / 245 G^{3}(d p)
$$

$G_{p s}^{\prime}$

the parameter of the $p-s$ interaction in the configuration $d^{n-1} s p$

$$
G_{p s}^{\prime}=1 / 3 G^{1}(p s)
$$

$\alpha, \alpha^{\prime}, \alpha^{\prime \prime}$

$\beta, \beta^{\prime}, \beta^{\prime \prime}$

$T, T^{\prime}, T^{\prime \prime}$

$H$

$H^{\prime}$

$J$

$J^{\prime}$

$K$

$K^{\prime}$

$G$

$\zeta_{d}, \zeta_{d}^{\prime}, \zeta_{d}^{\prime \prime}$

$\zeta_{p}, \zeta_{p}^{\prime}, \zeta_{p}^{\prime \prime}$

$\lambda, \lambda^{\prime}, \lambda^{\prime \prime}$

$\delta, \delta^{\prime}, \delta^{\prime \prime}$ correction parameters multiplying $L_{1}\left(L_{1}+1\right)$, where $L_{1}$ is the angular momentum of the core of $(d+s)^{n} p$, i.e., $(d+s)^{n}$

correction parameters multiplying the seniority operator of Racah

parameters of Trees multiplying the squared matrix of the interaction

$$
3 s^{2} 3 d^{n}-3 s 3 d^{n+1}
$$

parameter of the $d^{n}-d^{n-1} s$ interaction

$$
H=R^{2}(d d, d s) / 35
$$

parameter of the $d^{n-1} s-d^{n-2} s^{2}$ interaction, defined the same as $H$ parameter of the direct part of the $d^{n} p-d^{n-1} s p$ interaction,

$$
J=R^{2}(d p, s p) / 5
$$

parameter of the direct part of the $d^{n-1} s p-d^{n-2} s^{2} p$ interaction defined the same as $J$.

parameter of the exchange part of the $d^{n} p-d^{n-1} s p$ interaction,

$$
K=R^{1}(d p, p s) / 3
$$

parameter of the exchange part of the $d^{n-1} s p-d^{n-2} s^{2} p$ interaction defined the same as $K$.

parameter of the $d^{n} p-d^{n-2} s^{2} p$ interaction

$$
G=R^{2}(d d, s s) / 5=R^{2}(d s, s d) / 5=G^{2}(d s) / 5=G_{d s}^{\prime}
$$

parameters of the spin-orbit interaction of the $d$ electrons parameters of the spin-orbit interaction of the $p$ electron checking parameters multiplying $L(L+1)$, where $L$ is the net angular momentum for a particular term of $(d+s)^{n} p$

checking parameters multiplying $S(S+1)-S_{1}\left(S_{1}+1\right)$, where $S$ is the net spin of $(d+s)^{n} p$ and $S_{1}$ is the net spin of $(d+s)^{n}$.

The algebraic matrices of $(d+s)^{n} p$ were constructed and checked by the author with the purpose of using them to explain and predict the spectra of the configurations $(3 d+4 s)^{n} 4 p$ in neutral and singly ionized atoms of the iron group. The problem of constructing the matrices was considered previously, [11-13]. The purpose of the present paper is to describe a principal check for the algebraic matrices. The checked matrices of the configurations $(d+s)^{n} p$, for all permissible $n$, are available and can be obtained by request.

All checks are based upon the construction of such combinations of algebraic matrices so that the eigenvalues of the resulting matrix may be predicted theoretically. If we know the values 
of the parameters required in order to form the desired linear combinations, then we insert these values as coefficients multiplying the algebraic matrices found on tape, and diagonalize the resulting matrix. If the obtained eigenvalues differ from those predicted theoretically then it is usually not too difficult to find the reason for the discrepancy because in a particular check only a few of the algebraic matrices are utilized.

\section{Predicted Eigenvalues and Their Multiplicities}

As explained by Elliott [14] the eigenfunctions of a single particle which may be either in a $d$ shell or an $s$ shell can be considered as the basis of the representation (200) of the three dimensional unitary group $U_{3}$. The eigenfunctions of a system of $n$ such particles may be classified according to the irreducible representations of $U_{3}$ which appear in the reduction of the $n$th power of the representation (200). The electron $p$ can be considered as belonging to (100). We write these representations as $(p, q, r)$ where $p, q, r$ represent the lengths of the rows of the Young's tableau and $p \geqslant q \geqslant r$. For $(d+s)^{n}, p+q+r$ equals $2 n$, whereas for $(d+s)^{n} p, p+q+r$ equals $2 n+1$. The representations of $U_{3}$ which differ only by a number of columns of three blocks are equivalent representations of $S U_{3}$. Since $R_{3}$, whose representation is characterized by $L$ is a subgroup of $S U_{3}$, we can always subtract $r$ blocks from each row. The separation of the states belonging to the different representations may be made by an interaction whose eigenvalues are proportional to the Casimir operator of the group $S U_{3}$. If these eigenvalues are given by $g(p, q, r)$ we have the relationship

$$
g(p, q, r)=g(p-r, q-r, 0)
$$

Explicitly we have, [?5]

$$
g(p, q, r)=p^{2}+q^{2}+r^{2}-p q-p r-q r+3 p-3 r .
$$

The relationship (1) can also be checked directly from (2).

From Hamermesh [16], we obtain the rules of multiplying the Young's tableaux. From a paper by Racah [17] it is possible to obtain the values of $L$ which appear in each tableau. Table 1 gives the values of $L$ and the value of $2 g$ for each particular tableau.

\begin{tabular}{|c|c|c|c|c|c|}
\hline Representation & Values of $L$ & $2 \mathrm{~g}$ & Representation & Values of L & $2 \mathrm{~g}$ \\
\hline$(00)$ & S & 0 & $(52)$ & P D F F G H & 68 \\
\hline (10) & $\mathrm{P}$ & 8 & $(62)$ & S D D F G H I & 92 \\
\hline$(20)$ & S D & 20 & (72) & P D F F G H I K & 120 \\
\hline$(30)$ & P F & 36 & (33) & P F & 36 \\
\hline$(40)$ & S D G & 56 & $(43)$ & P D F G & 50 \\
\hline$(50)$ & P F H & 80 & (53) & P D F F G H & 68 \\
\hline$(60)$ & S D G I & 108 & (63) & P D F F G H I & 90 \\
\hline (70) & P F H K & 140 & (73) & P D F F G H H I K & 116 \\
\hline (11) & $\mathrm{P}$ & 8 & (44) & S D G & 56 \\
\hline (21) & P D & 18 & (54) & P D F G H & 72 \\
\hline (31) & P D F & 32 & (64) & S D D F G G H I & 92 \\
\hline$(41)$ & P D F G & 50 & (74) & P D F F G H H I K & 116 \\
\hline (51) & P D F G H & 72 & (55) & P F H & 80 \\
\hline (61) & P D F G H I & 98 & (65) & P D F G H I & 98 \\
\hline (71) & P D F G H I K & 128 & (75) & P D F F H H I K & 120 \\
\hline$(22)$ & S D & 20 & (66) & S D G I & 108 \\
\hline$(32)$ & P D F & 32 & (76) & P D F G H I K & 128 \\
\hline$(42)$ & S D D F G & 48 & (77) & P F H K & 140 \\
\hline
\end{tabular}

TABLE 1. Values of $\mathrm{L}$ appearing in the
representations of $\mathrm{SU}_{3}$ 
In order to obtain the tableaux of $(d+s)^{n}$, we consider two groups of electrons. Let the first group be of $(d+s)^{x}$, with all the electrons having $m_{s}$ equal to $1 / 2$ and thus $M_{s}$ equals $x / 2$. Let the second group be of $(d+s)^{n-x}$ with all the electrons having $m_{s}$ equal to $-1 / 2$ and thus $M_{s}$ equals $-(n-x) / 2$. In each group we use Pauli's principle by taking the tableau $(x)$ of maximal spin. Between the two groups Pauli's principle does not apply since they have different $m_{s}$. By multiplying the tableaux we get all the terms of $(d+s)^{n}$, with $M_{s}$ being equal to $(2 x-n) / 2$ and $S$ taking on the values between $n / 2$ and $|2 x-n| / 2$.

We now give the tableaux for $(d+s)^{n}$, and $(d+s)^{n} p$, where $n \leqslant 6$. The superscript on each tableau indicates the spin. Thus, the tableau of $(d+s)$ is written as ${ }^{2}(200)$.

In order to obtain the tableaux of $(d+s) p$, it is necessary to multiply the above tableau by ${ }^{2}(100)$. Then, $(d+s) p$ is given by

$$
{ }^{2}(200) \times{ }^{2}(100)=1,3(300)+{ }^{1,3}(210)
$$

For $(d+s)^{2}$ we have

$$
(200) \times(200)=(400)+(220)+(310) .
$$

By Pauli's principle we don't get singlets and triplets for each tableau. However, by a comparison with the known terms of $(d+s)^{2}$, we can write

$$
(d+s)^{2}: \quad{ }^{1}(400)+{ }^{1}(220)+{ }^{3}(310) .
$$

To obtain $(d+s)^{2} p$, we simply multiply the above by ${ }^{2}(100)$. Then,

$$
(d+s)^{2} p:{ }^{2}(500)+{ }^{2}(410)+{ }^{2}(320)+{ }^{2}(221)+{ }^{2,4}(410)+{ }^{2,4}(320)+{ }^{2,4}(311) .
$$

The tableaux of $(d+s)^{3}$ are obtained by multiplying (310), which is the tableau of highest spin in $(d+s)^{2}$, by (200). Again by comparing with the known terms of $(d+s)^{3}$ we can write

$$
\begin{aligned}
(d+s)^{3}: & { }^{2}(510)+{ }^{2}(420)+{ }^{2}(321)+{ }^{4}(411)+{ }^{4}(330) \\
(d+s)^{3} p: & { }^{1,3}(610)+{ }^{1,3}(520)+{ }^{1,3}(511)+1,3(520)+{ }^{1,3}(430)+{ }^{1,3}(421)+{ }^{1,3}(421) \\
& +{ }^{1,3}(331)+{ }^{1,3}(322)+{ }^{3,5}(511)+{ }^{3,5}(421)+{ }^{3,5}(430)+{ }^{3,5}(331) .
\end{aligned}
$$

To obtain the tableaux of $(d+s)^{4}$ we multiply (310) by (310). We note that in order to get only the triplets and quintets of $(d+s)^{4}$, we can multiply $(411)+(330)$, which are of highest spin in $(d+s)^{3}$, by (200). The latter multiplication is useful in helping to identify the spins of the various tableaux obtained by the multiplication of (310) by (310), which gives all the spins. Then by comparing with the known terms of $(d+s)^{4}$, we have

$$
\begin{aligned}
(d+s)^{4}: & { }^{1}(620)+{ }^{1}(440)+{ }^{1}(422)+{ }^{1}(521)+{ }^{3}(611)+{ }^{3}(530)+{ }^{3}(521)+{ }^{3}(431)+{ }^{3}(332) \\
& +{ }^{5}(431) \\
(d+s)^{4} p: & { }^{2}(720)+{ }^{2}(630)+{ }^{2}(621)+{ }^{2}(540)+{ }^{2}(441)+{ }^{2}(522)+{ }^{2}(432)+{ }^{2}(621) \\
& +{ }^{2}(531)+{ }^{2}(522)+{ }^{2,4}(711)+{ }^{2,4}(621)+{ }^{2,4}(630)+{ }^{2,4}(540)+{ }^{2,4}(531) \\
& +{ }^{2,4}(621)+{ }^{2,4}(531)+{ }^{2,4}(522)+{ }^{2,4}(531)+{ }^{2,4}(441)+{ }^{2,4}(432)+{ }^{2,4}(432) \\
& +{ }^{2,4}(333)+{ }^{4,6}(531)+{ }^{4,6}(441)+{ }^{4,6}(432) .
\end{aligned}
$$


To obtain the tableaux of $(d+s)^{5}$ we multiply $(411)+(330)$, which are of spin $3 / 2$ in $(d+s)^{3}$, by (310), which is of highest spin in $(d+s)^{2}$. Again, it is very useful to multiply (431), which is the tableau representing spin 2 in $(d+s)^{4}$, by (200). The latter result yields the quartets and sextets of $(d+s)^{5}$. By checking with the known terms of $(d+s)^{5}$ we have

$$
\begin{aligned}
(d+s)^{5}: & 2(721)+{ }^{2}(622)+{ }^{2}(640)+{ }^{2}(631)+{ }^{2}(541)+{ }^{2}(532)+{ }^{2}(442)+{ }^{4}(631) \\
& +{ }^{4}(541)+{ }^{4}(532)+{ }^{4}(433)+{ }^{6}(442) \\
(d+s)^{5} p: \quad & 1,3(821)+{ }^{1,3}(731)+1,3(722)+{ }^{1,3}(722)+{ }^{1,3}(632)+1,3(740)+{ }^{1,3}(650) \\
& +{ }^{1,3}(641)+{ }^{1,3}(731)+{ }^{1,3}(641)+{ }^{1,3}(632)+{ }^{1,3}(641)+{ }^{1,3}(551)+{ }^{1,3}(542) \\
& +{ }^{1,3}(632)+{ }^{1,3}(542)+{ }^{1,3}(533)+{ }^{1,3}(542)+1,3(443)+{ }^{3,5}(731)+{ }^{3,5}(641) \\
& +3,5(632)+{ }^{3,5}(641)+{ }^{3,5}(551)+{ }^{3,5}(542)+3,5(632)+3,5(542)+{ }^{3,5}(533) \\
& +{ }^{3,5}(533)+{ }^{3,5}(443)+{ }^{5,7}(542)+{ }^{5,7}(443) .
\end{aligned}
$$

The tableaux of $(d+s)^{6}$ are obtained by multiplying $(411)+(330)$, which are of highest spin in $(d+s)^{3}$, by $(411)+(330)$. If we multiply (442), which represents terms of spin $5 / 2$ in $(d+s)^{5}$, by (200), we obtain the quintets and septets of $(d+s)^{6}$. In addition, by multiplying (431), which gives the highest spin of $(d+s)^{4}$, by (310), we get the triplets, quintets and septets of $(d+s)^{6}$. The singlets are then what remains. To check we can again compare with the known terms of $(d+s)^{6}$. Thus

$$
\begin{aligned}
(d+s)^{6}: \quad & { }^{1}(822)+{ }^{1}(660)+{ }^{1}(741)+{ }^{1}(642)+{ }^{1}(642)+{ }^{1}(444)+{ }^{3}(741)+{ }^{3}(732) \\
& +{ }^{3}(642)+{ }^{3}(552)+{ }^{3}(633)+{ }^{3}(543)+{ }^{3}(651)+{ }^{5}(642)+{ }^{5}(543)+{ }^{7}(444) \\
(d+s)^{6} p: \quad & { }^{2}(922)+{ }^{2}(832)+{ }^{2}(760)+{ }^{2}(661)+{ }^{2}(841)+{ }^{2}(751)+{ }^{2}(742)+{ }^{2}(742) \\
& +{ }^{2}(652)+{ }^{2}(643)+{ }^{2}(742)+{ }^{2}(652)+{ }^{2}(643)+{ }^{2}(544)+{ }^{2,4}(841)+{ }^{2,4}(751) \\
& +{ }^{2,4}(742)+{ }^{2,4}(832)+{ }^{2,4}(742)+{ }^{2,4}(733)+{ }^{2,4}(742)+{ }^{2,}, 4(652)+{ }^{2,4}(643) \\
& +{ }^{2,4}(652)+{ }^{2,}(553)+{ }^{2,4}(733)+{ }^{2,4}(643)+{ }^{2,4}(643)+{ }^{2,4}(553)+{ }^{2,4}(544) \\
& +{ }^{2,4}(751)+{ }^{2,}, 4(661)+{ }^{2,4}(652)+{ }^{4,6}(742)+{ }^{4,6}(652)+{ }^{4,6}(643)+{ }^{4,6}(643) \\
& +{ }^{4,6}(553)+{ }^{4,6}(544)+{ }^{6,8}(544) .
\end{aligned}
$$

Tables 2-7 give the expected eigenvalues and their multiplicities for all terms of $(d+s)^{n} p$, where $n \leq 6$.

TABLE 2. Expected eigenvalues and their multiplicities for $(\mathrm{d}+\mathrm{s}) \mathrm{p}$

\begin{tabular}{l|c|c}
\hline \hline \multicolumn{1}{c|}{ TABLEAU } & $(30)$ & $(21)$ \\
\hline \multicolumn{1}{l|}{ Eigenvalue } & 36 & 18 \\
\hline Term & 1 & 1 \\
\hline${ }^{1} \mathrm{P}$ & 1 & 1 \\
${ }^{1} \mathrm{D}$ & 1 & 1 \\
${ }^{1} \mathrm{~F}$ & & 1 \\
${ }^{3} \mathrm{P}$ & 1 & \\
${ }^{3} \mathrm{D}$ & ${ }^{3} \mathrm{~F}$ & \\
\hline
\end{tabular}


TABLE 3. Expected eigenvalues and their multiplicities for $(\mathrm{d}+\mathrm{s})^{2} \mathrm{p}$

\begin{tabular}{|c|c|c|c|c|c|}
\hline TABLEAU & $(50)$ & (41) & (32) & (20) & (11) \\
\hline Term Eigenvalue & 80 & 50 & 32 & 20 & 8 \\
\hline${ }^{2} \mathrm{~S}$ & & & & 1 & \\
\hline${ }^{2} \mathrm{P}$ & 1 & 2 & 2 & & 1 \\
\hline${ }^{2} \mathrm{D}$ & & 2 & 2 & 1 & \\
\hline${ }^{2} \mathrm{~F}$ & 1 & 2 & 2 & & \\
\hline${ }^{2} \mathrm{G}$ & & 2 & & & \\
\hline${ }^{2} \mathrm{H}$ & 1 & & & & \\
\hline${ }^{4} \mathrm{~S}$ & & & & 1 & \\
\hline${ }^{4} \mathrm{P}$ & & 1 & 1 & & \\
\hline${ }^{4} \mathrm{D}$ & & 1 & 1 & 1 & \\
\hline${ }^{4} \mathrm{~F}$ & & 1 & 1 & & \\
\hline${ }^{4} \mathrm{G}$ & & 1 & - & & \\
\hline
\end{tabular}


TABLE 4. Expected eigenvalues and their multiplicities for $(\mathrm{d}+\mathrm{s})^{3} \mathrm{p}$

\begin{tabular}{|c|c|c|c|c|c|c|c|}
\hline TABLEAU & (61) & (52) & $(40)$ & (43) & (31) & (22) & (10) \\
\hline Eigenvalue & 98 & 68 & 56 & 50 & 32 & 20 & 8 \\
\hline \multicolumn{8}{|l|}{ Term } \\
\hline${ }^{1} \mathrm{~S}$ & & & 1 & & & 1 & \\
\hline${ }^{1} \mathrm{P}$ & 1 & 2 & & 1 & 2 & & 1 \\
\hline 'D & 1 & 2 & 1 & 1 & 2 & 1 & \\
\hline${ }^{1} \mathrm{~F}$ & 1 & 4 & & 1 & 2 & & \\
\hline${ }^{1} \mathrm{G}$ & 1 & 2 & 1 & 1 & & & \\
\hline${ }^{1} \mathrm{H}$ & 1 & 2 & & & & & \\
\hline${ }^{1} I$ & 1 & & & & & & \\
\hline${ }^{3} \mathrm{~S}$ & & & 2 & & & 2 & \\
\hline${ }^{3} \mathrm{P}$ & 1 & 2 & & 2 & 3 & & 1 \\
\hline${ }^{3} \mathrm{D}$ & 1 & 2 & 2 & 2 & 3 & 2 & \\
\hline${ }^{3} \mathrm{~F}$ & 1 & 4 & & 2 & 3 & & \\
\hline${ }^{3} \mathrm{G}$ & 1 & 2 & 2 & 2 & & & \\
\hline${ }^{3} \mathrm{H}$ & 1 & 2 & & & & & \\
\hline${ }^{3} \mathbf{I}$ & 1 & & & & & & \\
\hline${ }^{5} \mathrm{~S}$ & & & 1 & & & 1 & \\
\hline${ }^{5} \mathrm{P}$ & & & & 1 & 1 & & \\
\hline${ }^{5} \mathrm{D}$ & & & 1 & 1 & 1 & 1 & \\
\hline${ }^{5} \mathrm{~F}$ & & & & 1 & 1 & & \\
\hline${ }^{5} \mathrm{G}$ & & & 1 & 1 & & & \\
\hline
\end{tabular}


TABLE 5. Expected eigenvalues and their multiplicities for $(\mathrm{d}+\mathrm{s})^{4} \mathrm{p}$

\begin{tabular}{|c|c|c|c|c|c|c|c|c|}
\hline TABLEAU & (72) & (60) & (63) & (51) & (42) & (33) & (21) & (10) \\
\hline Term Eigenvalue & 120 & 108 & 90 & 72 & 48 & 36 & 18 & 0 \\
\hline${ }^{2} \mathrm{~S}$ & & 1 & & & 4 & & & 1 \\
\hline${ }^{2} \mathrm{P}$ & 1 & & 2 & 6 & & 5 & 3 & \\
\hline${ }^{2} \mathrm{D}$ & 1 & 1 & 2 & 6 & 8 & & 3 & \\
\hline${ }^{2} \mathrm{~F}$ & 2 & & 4 & 6 & 4 & 5 & & \\
\hline${ }^{2} \mathrm{G}$ & 1 & 1 & 4 & 6 & 4 & & & \\
\hline${ }^{2} \mathrm{H}$ & 2 & & 2 & 6 & & & & \\
\hline${ }^{2} \mathrm{I}$ & 1 & 1 & 2 & & & & & \\
\hline${ }^{2} \mathrm{~K}$ & 1 & & & & & & & \\
\hline${ }^{4} \mathrm{~S}$ & & 1 & & & 4 & & & 1 \\
\hline${ }^{4} \mathrm{P}$ & & & 1 & 3 & & 3 & 3 & \\
\hline${ }^{4} \mathrm{D}$ & & 1 & 1 & 3 & 8 & & 3 & \\
\hline${ }^{4} \mathrm{~F}$ & & & 2 & 3 & 4 & 3 & & \\
\hline${ }^{4} \mathrm{G}$ & & 1 & 2 & 3 & 4 & & & \\
\hline${ }^{4} \mathrm{H}$ & & & 1 & 3 & & & & \\
\hline${ }^{4} \mathrm{I}$ & & 1 & 1 & & & & & \\
\hline${ }^{6} \mathrm{~S}$ & & & & & 1 & & & \\
\hline${ }^{6} \mathrm{P}$ & & & & & & 1 & 1 & \\
\hline${ }^{6} \mathrm{D}$ & & & & & 2 & & 1 & \\
\hline${ }^{6} \mathrm{~F}$ & & & & & 1 & 1 & & \\
\hline${ }^{6} \mathrm{G}$ & & & & & 1 & & & \\
\hline
\end{tabular}


TABLE 6. Expected eigenvalues and their multiplicities for $(\mathrm{d}+\mathrm{s})^{5} \mathrm{p}$

\begin{tabular}{|c|c|c|c|c|c|c|c|c|c|c|c|}
\hline TABLEAU & (71) & (74) & (65) & (62) & (50) & (53) & (44) & (41) & (32) & (20) & (11) \\
\hline Eigenvalue & 128 & 116 & 98 & 92 & 80 & 68 & 56 & 50 & 32 & 20 & 8 \\
\hline 'S & & & & 2 & & & 1 & & & 1 & \\
\hline${ }^{1} \mathrm{P}$ & 1 & 1 & 1 & & 2 & 3 & & 3 & 3 & & 1 \\
\hline${ }^{1} \mathrm{D}$ & 1 & 1 & 1 & 4 & & 3 & 1 & 3 & 3 & 1 & \\
\hline${ }^{1} \mathrm{~F}$ & 1 & 2 & 1 & 2 & 2 & 6 & & 3 & 3 & & \\
\hline${ }^{1} \mathrm{G}$ & 1 & 2 & 1 & 4 & & 3 & 1 & 3 & & & \\
\hline${ }^{1} \mathrm{H}$ & 1 & 2 & 1 & 2 & 2 & 3 & & & & & \\
\hline${ }^{1} \mathrm{I}$ & 1 & 1 & 1 & 2 & & & & & & & \\
\hline${ }^{1} \mathrm{~K}$ & 1 & 1 & & & & & & & & & \\
\hline${ }^{3} \mathrm{~S}$ & & & & 3 & & & 2 & & & 3 & \\
\hline${ }^{3} \mathrm{P}$ & 1 & 1 & 1 & & 2 & 5 & & 5 & 5 & & 2 \\
\hline${ }^{3} \mathrm{D}$ & 1 & 1 & 1 & 6 & & 5 & 2 & 5 & 5 & 3 & \\
\hline${ }^{3} \mathrm{~F}$ & 1 & 2 & 1 & 3 & 2 & 10 & & 5 & 5 & & \\
\hline${ }^{3} \mathrm{G}$ & 1 & 2 & 1 & 6 & & 5 & 2 & 5 & & & \\
\hline${ }^{3} \mathrm{H}$ & 1 & 2 & 1 & 3 & 2 & 5 & & & & & \\
\hline${ }^{3} I$ & 1 & 1 & 1 & 3 & & & & & & & \\
\hline${ }^{3} \mathrm{~K}$ & 1 & 1 & & & & & & & & & \\
\hline${ }^{5} \mathrm{~S}$ & & & & 1 & & & 1 & & & 2 & \\
\hline${ }^{5} \mathrm{P}$ & & & & & & 2 & & 2 & 3 & & 2 \\
\hline${ }^{5} \mathrm{D}$ & & & & 2 & & 2 & 1 & 2 & 3 & 2 & \\
\hline${ }^{5} \mathrm{~F}$ & & & & 1 & & 4 & & 2 & 3 & & \\
\hline${ }^{5} \mathrm{G}$ & & & & 2 & & 2 & 1 & 2 & & & \\
\hline${ }^{5} \mathrm{H}$ & & & & 1 & & 2 & & & & & \\
\hline${ }^{5} \mathrm{I}$ & & & & 1 & & & & & & & \\
\hline${ }^{7} \mathrm{P}$ & & & & & & & & & 1 & & 1 \\
\hline${ }^{7} \mathrm{D}$ & & & & & & & & & 1 & & \\
\hline${ }^{7} \mathrm{~F}$ & & & & & & & & & 1 & & \\
\hline
\end{tabular}


TABLE 7. Expected eigenvalues and their multiplicities for $(\mathrm{d}+\mathrm{s})^{6} \mathrm{p}$

\begin{tabular}{|c|c|c|c|c|c|c|c|c|c|c|c|c|}
\hline TABLEAU & $(70)$ & $(76)$ & $(73)$ & (61) & (64) & (55) & (52) & $(40)$ & (43) & $(31)$ & $(22)$ & (10) \\
\hline $\begin{array}{l}\text { Eigenvalue } \\
\text { Term }\end{array}$ & 140 & 128 & 116 & 98 & 92 & 80 & 68 & 56 & 50 & 32 & 20 & 8 \\
\hline${ }^{2} \mathrm{~S}$ & & & & & 3 & & & 2 & & & 2 & \\
\hline${ }^{2} \mathrm{P}$ & 1 & 1 & 2 & 2 & & 2 & 6 & & 5 & 5 & & 2 \\
\hline${ }^{2} \mathrm{D}$ & & 1 & 2 & 2 & 6 & & 6 & 2 & 5 & 5 & 2 & \\
\hline${ }^{2} \mathrm{~F}$ & 1 & 1 & 4 & 2 & 3 & 2 & 12 & & 5 & 5 & & \\
\hline${ }^{2} \mathrm{G}$ & & 1 & 4 & 2 & 6 & & 6 & 2 & 5 & & & \\
\hline${ }^{2} \mathrm{H}$ & 1 & 1 & 4 & 2 & 3 & 2 & 6 & & & & & \\
\hline${ }^{2} \mathrm{I}$ & & 1 & 2 & 2 & 3 & & & & & & & \\
\hline${ }^{2} \mathrm{~K}$ & 1 & 1 & 2 & & & & & & & & & \\
\hline${ }^{4} \mathrm{~S}$ & & & & & 2 & & & 2 & & & 3 & \\
\hline${ }^{4} \mathrm{P}$ & & & 1 & 1 & & 1 & 4 & & 4 & 5 & & 2 \\
\hline${ }^{4} \mathrm{D}$ & & & 1 & 1 & 4 & & 4 & 2 & 4 & 5 & 3 & \\
\hline${ }^{4} \mathrm{~F}$ & & & 2 & 1 & 2 & 1 & 8 & & 4 & 5 & & \\
\hline${ }^{4} \mathrm{G}$ & & & 2 & 1 & 4 & & 4 & 2 & 4 & & & \\
\hline${ }^{4} \mathrm{H}$ & & & 2 & 1 & 2 & 1 & 4 & & & & & \\
\hline${ }^{4} \mathrm{I}$ & & & 1 & 1 & 2 & & & & & & & \\
\hline${ }^{4} \mathrm{~K}$ & & & 1 & & & & & & & & & \\
\hline${ }^{6} \mathrm{~S}$ & & & & & & & & & & & 1 & \\
\hline${ }^{6} \mathrm{P}$ & & & & & & & 1 & & 1 & 2 & & 2 \\
\hline${ }^{6} \mathrm{D}$ & & & & & & & 1 & & 1 & 2 & 1 & \\
\hline${ }^{6} \mathrm{~F}$ & & & & & & & 2 & & 1 & 2 & & \\
\hline${ }^{6} \mathrm{G}$ & & & & & & & 1 & & 1 & & & \\
\hline${ }^{6} \mathrm{H}$ & & & & & & & 1 & & & & & \\
\hline${ }^{8 P}$ & & & & & & & & & & & & 1 \\
\hline
\end{tabular}




\section{The Parameters of the Casimir Check}

Following Racah [18], we define the tensor operator $u^{(k)}$ by

Then let

$$
\left(n l\left\|u^{(k)}\right\| n^{\prime} l^{\prime}\right)=\delta_{n n^{\prime}} \delta_{l l^{\prime}}
$$

$$
U_{q}^{(k)}=\sum_{i=1}^{n} u_{q}^{(k)}[i]
$$

where $i$ refers to the $i$ th of $n$ electrons.

The group $S U_{2 l+1}$ is formed by the $4 l(l+1)$ tensor operators $U_{q}^{(k)}$ where $-k \leqslant q \leqslant k$ and $1 \leqslant k \leqslant 2 l$. Thus the group $S U_{3}$ is formed by the eight operators $U_{-1}^{(1)}, U_{0}^{(1)}, U_{1}^{(1)}, U_{-2}^{(2)}, U_{-1}^{(2)}, U_{0}^{(2)}, U_{1}^{(2)}$, and $U_{2}^{(2)}$. From equation (5-48) of Judd [19], we have for the Casimir operator of $S U_{3}$

$$
\begin{aligned}
G\left(S U_{3}\right) & =\frac{1}{6}\left\{3\left[U^{(1)}\right]^{2}+5\left[U^{(2)}\right]^{2}\right\} \\
& =\frac{1}{6}\left\{3\left[U^{(1)}\right]^{2}+5 \sum_{i=1}^{n}\left[u^{(2)}[i]\right]^{2}+10 \sum_{i<j} u^{(2)}[i] \cdot u^{(2)}[j]\right\} .
\end{aligned}
$$

From the above expression we can immediately obtain which parameters enter the Casimir check. From equation (103) of Racah [18], the first contribution is proportional to $L(L+1)$, and thus the parameter $\lambda$ must be included. The second term is a constant and is responsible for an additive term for each electron of a given $l$. The last contribution is a sum of products of tensors of second order and therefore only Slater parameters with $k$ equal to 2 enter the Casimir check. These are $F_{2}(d d)$ or $B, F_{2}(d p), G_{d s}^{\prime}, H$, and $J$.

If $E_{s}, E_{p}$, and $E_{d}$ are the additive constants for the electrons $s, p$, and $d$ respectively, then from table 1 ,

$$
\begin{aligned}
& { }^{2} \mathrm{~S}=E_{s}=20 \\
& { }^{2} \mathrm{P}=E_{p}+2 \lambda=8 \\
& { }^{2} \mathrm{D}=E_{d}+6 \lambda=20 .
\end{aligned}
$$

As the parameters $A$ and $C$ do not enter the Casimir check we have from (78) RII [1], the tableaux of $(d+s)^{2}$ and table 1 ,

$$
\begin{aligned}
& d^{2}{ }^{3} \mathrm{P}=2 E_{d}+7 B+2 \lambda=32 \\
& d^{2}{ }^{3} \mathrm{~F}=2 E_{d}-8 B+12 \lambda=32 \\
& d^{2}{ }^{1} \mathrm{G}=2 E_{d}+4 B+20 \lambda=56 .
\end{aligned}
$$

Solving (7), (8), and (9) yields

Then, from (5)

$$
E_{d}=11, \quad B=1, \quad \text { and } \lambda=\frac{3}{2} .
$$

$$
E_{p}=5
$$

From p. 197, [3] and the tableau for $d s^{3} \mathrm{D}$ we have

$$
d s^{3} \mathrm{D}=E_{d}+E_{s}-G_{d s}^{\prime}+6 \lambda=32 .
$$

Inserting the values for $E_{d}, E_{s}$, and $\lambda$ from (4) and (10) yields

$$
G_{d s}^{\prime}=8
$$


From (78) RII [1] and p. 197, [3]

$$
\begin{aligned}
& d s^{1} \mathrm{D}=E_{d}+E_{s}+G_{d s}^{\prime}+6 \lambda \\
& d^{2}{ }^{1} \mathrm{D}=2 E_{d}-3 B+6 \lambda .
\end{aligned}
$$

Then, from (4), (10), and (13), we have

$$
\begin{aligned}
& d s^{1} \mathrm{D}=48 \\
& d^{2}{ }^{1} \mathrm{D}=28 .
\end{aligned}
$$

Now, the interaction between $d^{2}{ }^{1} \mathrm{D}$ and $d s^{1} \mathrm{D}$ is given by $-2 \sqrt{35} H$, [20].

Then from (16) and (17), the eigenvalues of the matrix for ${ }^{1} \mathrm{D}$ are

$$
38 \pm \sqrt{100+140 H^{2}}
$$

Since, from the tableaux of $(d+s)^{2}$, and table 1, these eigenvalues should equal 20 and 56 , $H$ is given by

$$
H=\sqrt{40} / 5
$$

The parameter $G$ is the same as the parameter $G_{d s}^{\prime}[2]$, and thus should also have a value equal to 8 . This result can be checked in the following manner.

Using (78) RII and the tableaux of $(d+s)^{2}$ we have

$$
\begin{aligned}
& d^{2}{ }^{1} \mathrm{~S}=2 \boldsymbol{E}_{\boldsymbol{d}}+14 B=36 \\
& s^{2}{ }^{1} \mathrm{~S}=2 \boldsymbol{E}_{s}=40 .
\end{aligned}
$$

From (75) RIII the interaction between $d^{2}$ and $s^{2}$ is given by $\sqrt{5} G$. Thus, from (19) and (20), the eigenvalues of the matrix for ${ }^{1} \mathrm{~S}$ are $38 \pm \sqrt{4+5 G^{2}}$. Since the predicted eigenvalues are 20 and 56 , we obtain

$$
G=8 \text {. }
$$

From p. 200 TAS, the tableau for $d p^{1} \mathrm{~F}$ and table 1, we have

$$
d p^{1} \mathrm{~F}=E_{d}+E_{p}+2 F_{2}+12 \lambda=36 .
$$

By using (10) and (11)

Again from p. 200 TAS

$$
F_{2}=1 \text {. }
$$

$$
d p^{1} \mathrm{P}=E_{d}+E_{p}+7 F_{2}+2 \lambda .
$$

From (10), (11), and (22) we obtain

Similarly,

$$
d p^{1} \mathrm{P}=26 .
$$

$$
s p^{1} \mathrm{P}=E_{s}+E_{p}+2 \lambda=28 .
$$


By (7) Ref. [11], the interaction between $d p^{1} \mathrm{P}$ and $s p^{1} \mathrm{P}$ is given by $-(J+K) \sqrt{2}$.

As $K$ is a Slater parameter with $k$ equal to 1 it does not enter the Casimir check. Then from (24) and (25), the eigenvalues of ${ }^{1} \mathrm{P}$ are given by

$$
27 \pm \sqrt{1+2 J^{2}}
$$

Since the predicted eigenvalues are 18 and 36 , we obtain

$$
J=2 \sqrt{10}
$$

If we now consider $(d+s)^{n} p$ we have

$$
\begin{gathered}
A\left(d^{n} p\right)=n E_{d}+E_{p} \\
=11 n+5 \\
S^{\prime}=A^{\prime}\left(d^{n-1} s p\right)-A\left(d^{n} p\right) \\
=(n-1) E_{d}+E_{s}+E_{p}-n E_{d}-E_{p} \\
=E_{s}-E_{d}=9 .
\end{gathered}
$$

In the configuration $d^{n-2} s^{2} p$ we must also take into account the exchange interaction between the electrons $d$ and $s$ which is given by $-(n-2) G_{d s}^{\prime}$, [11]. This is a constant for each term of $d^{n-2} s^{2} p$ and thus the matrix multiplying $G_{d s}^{\prime}$ is not put on tape, but must be added to the height of the configuration.

Thus,

$$
\begin{aligned}
S^{\prime \prime} & =A^{\prime \prime}\left(d^{n-2} s^{2} p\right)-A\left(d^{n} p\right) \\
& =(n-2) E_{d}+2 E_{s}+E_{p}-(n-2) G_{d s}-n E_{d}-E_{p} \\
& =-2 E_{d}+2 E_{s}-(n-2) 8 \\
& =34-8 n .
\end{aligned}
$$

Thus, we have the following parameters for the Casimir check of $(d+s)^{n}$ :

$$
\begin{aligned}
A & =11 n+5 \\
S^{\prime} & =9 \\
S^{\prime \prime} & =34-8 n \\
B & =B^{\prime}=B^{\prime \prime}=1 \\
G_{d s}^{\prime} & =G=8 \\
F_{2} & =F_{2}^{\prime}=F_{2}^{\prime \prime}=1 \\
H & =H^{\prime}=\sqrt{40} / 5 \\
J & =J^{\prime}=2 \sqrt{10} \\
\lambda & =\lambda^{\prime}=\lambda^{\prime \prime}=\frac{3}{2} .
\end{aligned}
$$

The other parameters equal zero.

If we take into consideration only the parameters which enter the Casimir check, we have from eqs (1) and (2) p. 296 TAS and eqs (11) and (12) pp. 182, 183 TAS, that the difference between the configurations $d^{6} p$ and $d^{4} p$ is given by

$$
-(25 / 2) \mathrm{C}_{222} F^{2}(d d)-[(-4 \times 5) / 2] \mathrm{C}_{222} F^{2}(d d) .
$$


By eq (9) p. 182, TAS, the above expression reduces to $-(2 / 7) F^{2}(d, d)$ which equals $-14 B$ here. Since the matrices of $B$ for $d^{6} p$ which we used are the same as those of $d^{4} p$, the above contribution must be incorporated into the height of the configuration $d^{6} p$.

Then, for $(d+s)^{6} p$

$$
\begin{aligned}
A\left(d^{6} p\right) & =6 E_{d}+E_{p}-14 B=66+5-14=57 \\
A^{\prime}\left(d^{5} s p\right) & =5 E_{d}+E_{s}+E_{p}=80 \\
S^{\prime} & =80-57=23 \\
A^{\prime \prime}\left(d^{4} s^{2} p\right) & =4 E_{d}+2 E_{s}+E_{p}-4 G_{d s}^{\prime} \\
& =57 \\
S^{\prime \prime} & =0 .
\end{aligned}
$$

\section{The Casimir Check for $(d+s)^{n} p, n>6$}

The configuration $(d+s)^{12}$ consists of the single term $d^{10} s^{2}{ }^{1} \mathrm{~S}$. In this case $p+q+r$ equals 24 , and thus the Young's tableau for $(d+s)^{12}$ must be $(8,8,8)$. Now, $(d+s)^{n}$ and $(d+s)^{12-n}$ together form $(d+s)^{12}$. Thus, if the Young's tableaux of $(d+s)^{12-n}$ are $(p, q, r)$, then the tableaux for $(d+s)^{n}$ are $(8-r, 8-q, 8-p)$ since in $(p, q, r), r$ has the smallest number of blocks so that $(8-r)$ has the largest number. The configuration $(d+s)^{6}$ is self-complementary. For $(d+s)^{n} p$, the $p$ electron is then added to any row as long as $p \geqslant q \geqslant r$.

In Ref. [11] it was shown that for $n>6$, it is only necessary to calculate the matrices of the parameters $G_{1}^{\prime}, G_{3}^{\prime}, K$, and $K^{\prime}$. However, none of these parameters enter the Casimir check and thus we only need this check for $n \leqslant 6$. It is, nevertheless, instructive to obtain the Young's tableaux for at least one case when $n>6$. Thus, we consider the configuration $(d+s)^{10} p$. Since the tableaux of $(d+s)^{2}$ are

$$
{ }^{1}(40)+{ }^{1}(22)+{ }^{3}(31)
$$

the tableaux of $(d+s)^{10}$ are

$$
{ }^{1}(884)+{ }^{1}(866)+{ }^{3}(875)
$$

We note that the expected eigenvalues are the same as those of $(d+s)^{2}$. This is generally true because the only difference between $(d+s)^{n}$ and $(d+s)^{12-n}$ is a change in sign of the matrices of $H$, for $n$ even. However, changing the sign of all the matrix elements of the interactions $d^{n}-d^{n-1} s$, and $d^{n-1} s-d^{n-2} s^{2}$ is equivalent to changing the sign of the rows and columns of $d^{n-1} s$. This transformation evidently does not change the eigenvalues. That the eigenvalues of the Casimir check for the configurations $(d+s)^{n}$ and $(d+s)^{12-n}$ are the same can also be seen from (2), as generally

$$
g(p, q, r)=g(a-r, a-q, a-p) .
$$

Thus, specifically, for $(d+s)^{n}$ and $(d+s)^{12-n}$

$$
g(p, q, r)=g(8-r, 8-q, 8-p) .
$$

However, the tableaux of $(d+s){ }^{10} p$ are

$$
{ }^{2}(54)+{ }^{2}(33)+{ }^{2}(30)+{ }^{2}(21)+{ }^{2,4}(42)+{ }^{2,4}(33)+{ }^{2,4}(21) .
$$

We note that the expected eigenvalues differ from those of $(d+s)^{2} p$. This is because when going from $(d+s)^{n} p$ to $(d+s)^{12-n} p$ the matrices of the parameters $F_{2}, F_{2}^{\prime}$, and $\mathrm{F}_{2}^{\prime \prime}$ change sign. This change in sign affects the diagonal elements also and is sufficient to cause the eigenvalues to change. 
In addition, the matrices of either $J$ and $J^{\prime}$ or $H$ and $H^{\prime}$ change sign as we go from $(d+s)^{n} p$ to $(d+s)^{12-n} p$. Thus, in the Casimir check not only are the matrix elements of the interaction between configurations in $(d+s)^{n} p$ of different sign than those of $(d+s)^{12-n} p$, but they also differ in magnitude.

\section{References}

[1] Racah, G., Phys. Rev. 62, 438 (1942), referred to as R II.

[2] Racah, G., Phys. Rev. 63, 367 (1943), referred to as R III.

[3] Condon, E. U., and Shortley, G. H., The Theory of Atomic Spectra (Cambridge University Press, 1935), referred to as TAS.

[4] Trees, R. E., Phys. Rev. 83, 756 (1951); ibd., 84, 1089 (1951).

[5] Racah, G., Phys. Rev. 85, 381 (1952).

[6] Racah, G., Rydeberg Centennial Conference, Acta Univ. Lund. 50,31 (1955).

[7] Racah, G., and Shadmi, Y., Phys. Rev. 119, 156 (1960).

[8] Trees, R. E., and Jorgensen, C. K., Phys. Rev. 123, 1278 (1961).

[9] Trees, R. E., Phys. Rev. 129, 1220 (1963).

[10] Rosenzweig, N., Phys. Rev. 88, 580 (1952).

[11] Roth, C., J. Math. Phys. 10, No. 6, 1038 (1969).
[12] Roth, C., J. Math. Phys. 9, No. 4, 686 (1968).

[13] Roth, C., J. Math. Phys. 9, 1832 (1968).

$14]$ Elliott, J. P., Proc. Roy. Soc. A245, 128 (1958).

15] Racah, G., Bull. Res. Counc. Israel, 8F, No. 1, 1 (1959).

[16] Hamermesh, M., Group Theory and Its Application to Physical Problems (Addison-Wesley, 1962), Chapter 7.

[17] Racah, G., Rev. Mod. Phys. 21, No. 3, 494 (1949).

[18] Racah, G., Group Theory and Spectroscopy, Mimeographed Notes (Princeton 1951. Published, CERN, Geneva, 1961).

[19] Judd, B. R., Operator Techniques in Atomic Spectroscopy (McGraw-Hill, 1963).

[20] Fano, U., and Racah, G., Irreducible Tensorial Sets (Academic Press, 1959), Chapter 16.

(Paper 75B1\&2-342) 